\title{
Local structure of dilute aqueous DMSO solutions, as seen from molecular dynamics simulations
}

Abdenacer Idrissi, Bogdan A. Marekha, Mohammed Barj, François Alexandre Miannay, Toshiyuki Takamuku, Vasilios Raptis, Jannis Samios, and Pál Jedlovszky

Citation: The Journal of Chemical Physics 146, 234507 (2017); doi: 10.1063/1.4985630

View online: http://dx.doi.org/10.1063/1.4985630

View Table of Contents: http://aip.scitation.org/toc/jcp/146/23

Published by the American Institute of Physics

\section{Articles you may be interested in}

Water-anion hydrogen bonding dynamics: Ultrafast IR experiments and simulations

The Journal of Chemical Physics 146, 234501 (2017); 10.1063/1.4984766

The entropy of a complex molecule

The Journal of Chemical Physics 146, 224106 (2017); 10.1063/1.4984965

Water structure around hydrophobic amino acid side chain analogs using different water models

The Journal of Chemical Physics 146, 225104 (2017); 10.1063/1.4985671

Liquid-liquid phase transition in an ionic model of silica

The Journal of Chemical Physics 146, 234503 (2017); 10.1063/1.4984335

Structure and mobility of water confined in $\mathrm{AlPO}_{4}-54$ nanotubes

The Journal of Chemical Physics 146, 234509 (2017); 10.1063/1.4985626

Water flow in carbon nanotubes: The effect of tube flexibility and thermostat

The Journal of Chemical Physics 146, 234701 (2017); 10.1063/1.4985252

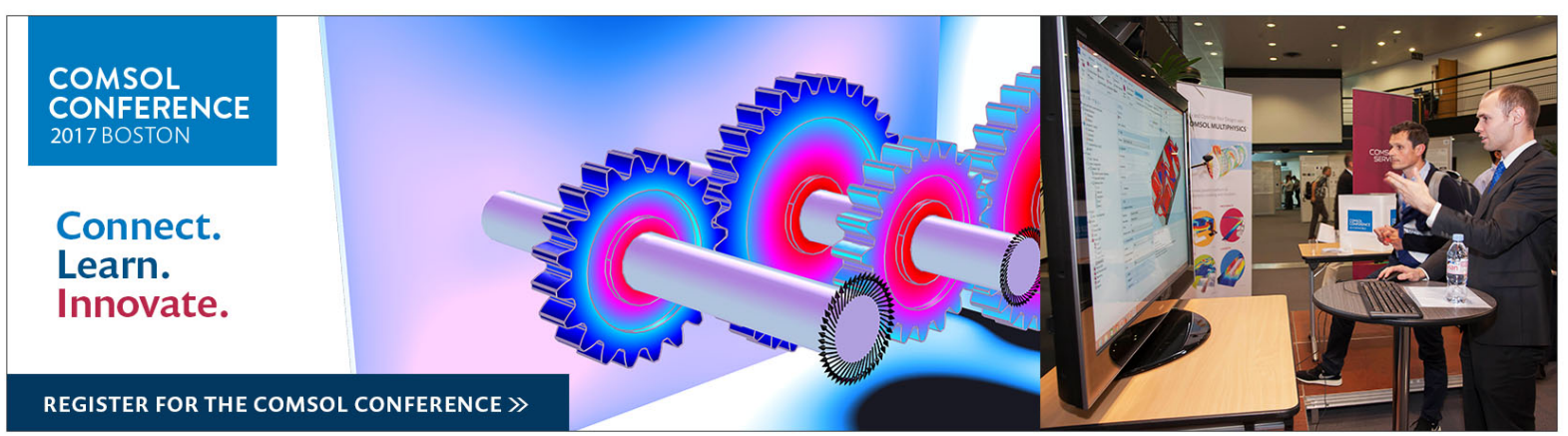




\title{
Local structure of dilute aqueous DMSO solutions, as seen from molecular dynamics simulations
}

\author{
Abdenacer Idrissi, ${ }^{1, a)}$ Bogdan A. Marekha, ${ }^{2}$ Mohammed Barj, ${ }^{1}$ \\ François Alexandre Miannay, ${ }^{1}$ Toshiyuki Takamuku, ${ }^{3}$ Vasilios Raptis, ${ }^{4,5}$ \\ Jannis Samios, ${ }^{6}$ and Pál Jedlovszky ${ }^{7,8,9}$ \\ ${ }^{1}$ University of Lille, Science and Technology, LASIR (UMR CNRS A8516), Bât. C5, Cité Scientifique, \\ 59655 Villeneuve d'Ascq Cedex, France \\ ${ }^{2}$ Normandie Université, UNICAEN, CERMN (Center d'Etudes et de Recherche sur le Médicament \\ de Normandie)-FR CNRS INC3M, Boulevard Becquerel, 14032 Caen Cedex, France \\ ${ }^{3}$ Department of Chemistry and Applied Chemistry, Graduate School of Science and Engineering, Saga University, \\ Honjo-machi, Saga 840-8502, Japan \\ ${ }^{4}$ Department of Chemical Engineering, Center for Process Systems Engineering, Molecular Systems Engineering \\ Group, Imperial College London, South Kensington Campus, London SW7 2AZ, England, United Kingdom \\ ${ }^{5}$ Institute of Molecular Biology and Biotechnology, Foundation for Research and Technology-Hellas, \\ Heraklion 700-13, Greece \\ ${ }^{6}$ Laboratory of Physical Chemistry, Department of Chemistry, University of Athens, Panepistimiopolis, \\ Athens 157-71, Greece \\ ${ }^{7}$ Department of Chemistry, Eszterházy Károly University, Leányka utca 6, H-3300 Eger, Hungary \\ ${ }^{8}$ MTA-BME Research Group of Technical Analytical Chemistry, Szt. Gellért tér 4, H-1111 Budapest, Hungary \\ ${ }^{9}$ Laboratory of Interfaces and Nanosize Systems, Institute of Chemistry, Eötvös Loránd University, \\ Pázmány P. Stny 1/A, H-1117 Budapest, Hungary
}

(Received 13 January 2017; accepted 30 May 2017; published online 19 June 2017)

The information about the structure of dimethyl sulfoxide (DMSO)-water mixtures at relatively low DMSO mole fractions is an important step in order to understand their cryoprotective properties as well as the solvation process of proteins and amino acids. Classical MD simulations, using the potential model combination that best reproduces the free energy of mixing of these compounds, are used to analyze the local structure of DMSO-water mixtures at DMSO mole fractions below 0.2. Significant changes in the local structure of DMSO are observed around the DMSO mole fraction of 0.1. The array of evidence, based on the cluster and the metric and topological parameters of the Voronoi polyhedra distributions, indicates that these changes are associated with the simultaneous increase of the number of DMSO-water and decrease of water-water hydrogen bonds with increasing DMSO concentration. The inversion between the dominance of these two types of H-bonds occurs around $X_{\text {DMSO }}=0.1$, above which the DMSO-DMSO interactions also start playing an important role. In other words, below the DMSO mole fraction of 0.1, DMSO molecules are mainly solvated by water molecules, while above it, their solvation shell consists of a mixture of water and DMSO. The trigonal, tetrahedral, and trigonal bipyramidal distributions of water shift to lower corresponding order parameter values indicating the loosening of these orientations. Adding DMSO does not affect the hydrogen bonding between a reference water molecule and its first neighbor hydrogen bonded water molecules, while it increases the bent hydrogen bond geometry involving the second ones. The close-packed local structure of the third, fourth, and fifth water neighbors also is reinforced. In accordance with previous theoretical and experimental data, the hydrogen bonding between water and the first, the second, and the third DMSO neighbors is stronger than that with its corresponding water neighbors. At a given DMSO mole fraction, the behavior of the intensity of the high orientational order parameter values indicates that water molecules are more ordered in the vicinity of the hydrophilic group while their structure is close-packed near the hydrophobic group of DMSO. Published by AIP Publishing. [http://dx.doi.org/10.1063/1.4985630]

\section{INTRODUCTION}

Low mole fraction dimethyl sulfoxide (DMSO)-water mixtures, below the DMSO mole fraction $X_{\mathrm{DMSO}}=0.13$, are

\footnotetext{
a) Author to whom correspondence should be addressed: nacer.idrissi@univlille1.fr
}

commonly employed as cryoprotective agents of proteins and phospholipid bilayers. Moreover, the solvation dynamics of solutes, such as cellulose, ${ }^{1}$ proteins, ${ }^{2}$ enzymes,${ }^{3}$ and amino acids, ${ }^{4}$ depends strongly on the change of the local structure of the DMSO-water mixture as a function of $X_{\text {DMSO }}$. Indeed, Fig. 4 of Ref. 1 shows that the adsorption of both DMSO and water on cellulose moderately increases in the range of $X_{\text {DMSO }}$ $<0.1$, the adsorption for water being noticeably larger than that 
for DMSO, whereas in the range $0.1<X_{\text {DMSO }}<0.2$, the adsorption of DMSO becomes considerably higher, while water desorbs from the cellulose surface. ${ }^{1}$ The analysis of the solvation of proteins, such as ribonuclease, lysozyme, $\beta$-lactoglobulin, and chymotrypsinogen, in DMSO/water mixtures shows that a transition occurs from preferential hydration at low DMSO mole fractions $\left(X_{\text {DMSO }}<0.13\right)$ to preferential DMSO binding at higher DMSO mole fractions. ${ }^{2}$ In another work, the solvation of three amino acids, namely, glycine, $\mathrm{L}$-alanine, and L-serine, was analyzed in DMSO-water mixtures in the composition range of $0.1<X_{\text {DMSO }}<0.25 .^{4}$ The results demonstrated that the interaction between amino acids and DMSO is strongly correlated with the change of the local structure of the mixture as a function of $X_{\mathrm{DMSO}}$. These results pointed out that the analysis of the structure of DMSO-water mixtures at low DMSO mole fractions is of high interest, in order to elucidate how the local structure in these mixtures can be correlated with the solvation dynamics of the above-mentioned biomolecules. Indeed, several physical-chemical properties undergo a complex change in the low $X_{\text {DMSO }}$ (water-rich) region. ${ }^{5}$ At the molecular level, these properties are manifestations of the DMSO-DMSO, DMSO-water, and water-water intermolecular interactions, which are associated with important processes that occur in the aqueous mixture, such as self-association (aggregation, clustering, etc.), solvation, and the changes of the local structure of water to accommodate the dissolution of the solute. Concerning the latter process, it is important to understand how the local structure of water is affected by the addition of DMSO molecules that possess both hydrophobic $\left(\mathrm{CH}_{3}\right)$ and hydrophilic (SO) groups. The variation of the mixture mole fraction is an easy way of modulating these interactions. Even more interestingly, the quantities that are accurately measured in small increments of the mixture composition reveal new aspects on the intermolecular interactions in the mixture. ${ }^{6,7}$

However, as pointed out in an earlier work, ${ }^{5}$ the interpretation of experimental data at a low DMSO mole fraction range is controversial, particularly on the effect of DMSO on the structure of water and on the extent of the self-association of DMSO. Indeed, the interpretation of the thermodynamic, dielectric, and spectroscopic measurements leads to the conclusion that adding a small amount of DMSO to water is accompanied by the concomitant formation of highly polar aggregates (strong dipole-dipole interactions or self-association) and the breakdown of the water structure. ${ }^{8}$ In another study, conducted in the range of DMSO mole fractions between 0 and 0.13 concerning the effect of DMSO on the temperature of maximum density (TMD) of water, it was concluded that DMSO destabilizes the structure of water. ${ }^{9}$ On the other hand, thermodynamic data based on densities and molar heat capacities measured at low DMSO mole fractions suggest that the structure of water is enhanced by the presence of a small amount of DMSO. ${ }^{10,11}$ The same conclusion was reached using infrared (IR) spectroscopy ${ }^{12}$ and X-ray diffraction ${ }^{13}$ measurements as well. Furthermore, the surface tension of the DMSO-water mixtures decreases rapidly in dilute solutions (below $X_{\text {DMSO }}$ $=0.2$ ) but becomes almost constant upon further increase of $X_{\text {DMSO }}{ }^{14}$ In another IR spectroscopy study,,${ }^{15}$ it was revealed that the positions of the $\mathrm{C}-\mathrm{H}$ and $\mathrm{O}-\mathrm{H}$ vibrational modes exhibit a nonlinear behavior, in particular, in the $X_{\text {DMSO }}$ range below 0.1 . The $\mathrm{C}-\mathrm{H}$ vibrational mode is slightly affected and undergoes a red shift on further increase of $X_{\text {DMSO }}$ (see Fig. 7 of Ref. 15). Mizuno et al. reported ${ }^{16}$ that in the water-rich region, the $\mathrm{C}-\mathrm{H}$ and $\mathrm{O}-\mathrm{H}$ vibrational modes as well as their corresponding ${ }^{1} \mathrm{H}$ chemical shifts vary in a non-linear manner (see Figs. 2 and 3 in Ref. 16). Using dielectric relaxation spectroscopy, a breakdown of the water structure was observed in dilute DMSO aqueous solutions. ${ }^{17}$ In a femtosecond IR study of the dynamics of water molecules in water-acetone and water-DMSO mixtures, it was shown that the rate of increase of the normalized ratio for the amplitude of the slow water molecules is high in the range of $X_{\text {DMSO }}$ between 0 and 0.08

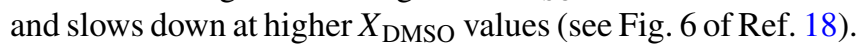
This was assigned to the weak tendency of DMSO molecules to self-associate. ${ }^{18}$ The intermolecular interactions between DMSO and water were investigated with high-resolution $\mathrm{X}$-ray absorption, emission, and inelastic spectroscopy using the micro-jet technique. ${ }^{19}$ The results showed that DMSO molecules distort the hydrogen bond network of water already in water-rich systems. Mass spectroscopy analysis of the clusters in DMSO-water mixtures ${ }^{20}$ showed that the clustering of DMSO or water molecules is non-linearly dependent on $X_{\text {DMSO }}$, exhibiting the existence of a critical value of $X_{\text {DMSO }}$ around 0.10 . Indeed, it was shown that DMSO is preferentially solvated by water below $X_{\mathrm{DMSO}} \sim 0.10$, while upon further increase of the DMSO concentration it becomes preferentially solvated by other DMSO molecules. These conclusions were confirmed by the static light scattering experiment of Huang et al. ${ }^{4}$

Computer simulations can help understanding the microscopic mechanisms underlying the above phenomena. For this purpose, a variety of models have been investigated in order to reproduce the properties of water/DMSO mixtures. For instance, Chalaris and Samios ${ }^{21}$ used a number of available potentials for water and DMSO to simulate mixtures at DMSO mole fractions between 0.055 and 0.67 at $298 \mathrm{~K}$ at densities corresponding to the atmospheric pressure. They found that the combination of the Single Point Charge (SPC) model ${ }^{22}$ of water and P2 model of DMSO developed by Luzar and Chandler $^{23}$ gives the most reliable description of the mixture in terms of mean potential energy per mole as well as of pressure and self-diffusivity of the two components, but further adjustments are needed to improve the agreement with experimental data. Mancera et $_{\text {al. }}{ }^{24}$ simulated dilute $\left(X_{\mathrm{DMSO}}=0.055\right)$ aqueous solutions of DMSO at 298, 318, and $338 \mathrm{~K}$ using the TIP4P model $^{25}$ for water and P2 model for DMSO. They found that the DMSO molecules are strongly hydrated, forming hydrogen bonds with neighboring water molecules involving the DMSO oxygen atoms, whereas methyl groups are hydrophobically hydrated by ordered aquatic shells. In both cases, an increased lifetime of water-water hydrogen bonds formed around the DMSO molecules was observed. This enhancement effect on the local structure and dynamics of water around DMSO was found to be more pronounced at higher DMSO concentrations in the simulation study of Bopp et al., performed at $X_{\text {DMSO }}$ $=0.055$ and 0.19 at $298 \mathrm{~K}^{26}$ Furthermore, using molecular dynamics (MD) simulations with the GROMOS model of 
$\mathrm{DMSO}^{27}$ and the SPC/E model of water, ${ }^{28}$ Roy et al. ${ }^{29}$ found a percolation threshold around the $X_{\text {DMSO }}$ value of $0.12-0.15$, below which a spanning cluster is formed, comprising DMSO: $2 \mathrm{H}_{2} \mathrm{O}$ species through methyl-methyl aggregation and hydrogen bonding.

The choice of the proper potential models of the components is an important issue of simulations of mixtures. ${ }^{30}$ Indeed, although the intermolecular potentials of liquid water and DMSO could be individually capable of reproducing the structure and dynamics of the respective neat liquids, their combination does not necessarily reproduce the structure and dynamics of their mixtures. The free energy of mixing of the two components provides a rigorous test of the force field accuracy; the good reproduction of its experimental value at various compositions is a pre-requisite of the accurate description of the microscopic structure (e.g., in terms of self-association) of the mixture..$^{31-34}$ Our recent study on the DMSO-water mixtures showed that the combination of the DMSO model proposed earlier by Vishnyakov, Laaksonen, and Lyubartsev $(\mathrm{VLL})^{35}$ and the TIP4P $\mathrm{P}^{25}$ potential of water gives the best agreement with the experimental free energy of mixing data. ${ }^{36}$ We used this potential model combination afterwards in a set of MD simulations of DMSO-water mixtures of various compositions and analyzed in detail the resulting structure by means of the Voronoi polyhedra (VP). ${ }^{37}$ We found that the dilution of the two neat liquids by the other component follows different mechanisms, which stems from the fact that the tendency of DMSO molecules to be in contact with water molecules is clearly stronger than that of water molecules to be in contact with DMSO. We have demonstrated that neither of the two molecules tends to form relatively large self-associates over the entire range of compositions, in a clear contrast with entropy driven mixtures, such as the methanol-acetone system. ${ }^{38,39}$

The aim of the present paper is to use MD simulations to analyze the local structure in DMSO-water mixtures in the DMSO mole fraction range between 0.0 and 0.20 , using a small increment of $X_{\text {DMSO }}$ of 0.02 . Our main goal is to quantify the extent of the self-association of DMSO and the effect of DMSO on the local structure of water. For this purpose, we calculate statistical functions over a small volume around the hydrophilic $(\mathrm{S}=\mathrm{O})$ and hydrophobic $\left(\mathrm{CH}_{3}\right)$ groups of DMSO and around the $\mathrm{O}-\mathrm{H}$ group of water. The local structure around DMSO molecules was analyzed using the cluster distribution of DMSO and the metric and topological characteristics of the VP, while the effect of DMSO on the structure of water was analyzed by calculating three order parameter distributions, describing the trigonal, tetrahedral, and trigonal bipyramidal local structures of water. Two hydrogen bonding characteristic distances were proposed to quantify the effect of DMSO on the hydrogen bonding between water molecules and between water and hydrophilic and hydrophobic groups of DMSO.

The paper is organized as follows. In Sec. II details on the simulation performed are provided. In Sec. III the definitions and properties of the statistical functions used in this work to characterize unambiguously the local structure are summarized, whereas in Sec. IV the results of this study are presented and discussed in detail.
Finally, in Sec. V the main conclusions of this study are summarized.

\section{COMPUTATIONAL DETAILS}

The potential parameters for both models consist of a pairwise sum of site-site interactions, described by a sum of Coulombic and Lennard-Jones terms, in the form

$$
\mathrm{u}_{\mathrm{ij}}=\sum_{\mathrm{a}} \sum_{\mathrm{b}} \frac{1}{4 \pi \varepsilon_{0}} \frac{\mathrm{q}_{\mathrm{a}} \mathrm{q}_{\mathrm{b}}}{\mathrm{r}_{\mathrm{ia}, \mathrm{jb}}}+4 \varepsilon_{\mathrm{ab}}\left[\left(\frac{\sigma_{\mathrm{ab}}}{\mathrm{r}_{\mathrm{i} a, \mathrm{~b}}}\right)^{12}-\left(\frac{\sigma_{\mathrm{ab}}}{\mathrm{r}_{\mathrm{i} a, j \mathrm{~b}}}\right)^{6}\right],
$$

where indices $a$ and $b$ run over the interaction sites of molecules $i$ and $j$, respectively, $r_{i a, j b}$ is the distance of site $a$ of molecule $i$ from site $b$ of molecule $j, \varepsilon_{a b}$ and $\sigma_{a b}$ are the Lennard-Jones energy and distance parameters, respectively, $q_{a}$ and $q_{b}$ denote the fractional charges carried by the corresponding sites, and $\varepsilon_{0}$ is the vacuum permittivity. In the VLL model, the $\mathrm{CH}_{3}$ groups of the DMSO molecule are treated as united atoms (i.e., as single interaction sites), whereas in the TIP4P water model, the negative fractional charge is displaced from the $\mathrm{O}$ atom by $0.15 \AA$ along the bisector of the $\mathrm{H}-\mathrm{O}-\mathrm{H}$ angle. Crossed interactions were computed through the Lorentz-Berthelot rules, i.e., $\varepsilon_{a b}=\left(\varepsilon_{a} \varepsilon_{b}\right)^{\frac{1}{2}}$ and $\sigma_{a b}=\frac{1}{2}\left(\sigma_{a}+\sigma_{b}\right)$. The intermolecular interaction parameters are given, among others, in our previous publications. ${ }^{36,37}$ The simulations have been performed using the DL_POLY program. ${ }^{40}$ All the simulations have been carried out on the isothermal-isobaric $(N P T)$ ensemble at $1 \mathrm{~atm}$ and $298 \mathrm{~K}$. The weak coupling algorithms of Berendsen et al. ${ }^{41}$ were used with barostat and thermostat relaxation times of 0.1 and $0.2 \mathrm{ps}$, respectively. Long range electrostatic interactions have been treated with the Ewald summation technique. ${ }^{42,43}$ The simulations have been carried out using a cutoff radius equal to half the simulation box length, for the nonbonded interactions. The local structure of DMSO/water mixtures have been studied in the DMSO mole fraction range between 0.0 and 0.20 , using a small increment of 0.02 . The pure components have also been simulated to get information on their local structures. As our main objective is to characterize the local structure at a low mole fraction of DMSO, a total number of 2048 molecules have been used. The number of DMSO molecules in the studied systems has been $40,82,123,165$, 206, 246, 288, 328, 369, and 411, respectively. This allows us to calculate the relevant distribution functions associated with the position of the DMSO molecules with a reasonable statistical accuracy. The equations-of-motion have been solved using the Verlet leapfrog integration algorithm, with an integration time step of 2 fs. The systems have been equilibrated for $5 \mathrm{~ns}$. Production runs of 1 ns have been performed afterwards, to produce $10^{4}$ saved sample configurations, separated by 0.1 ps long trajectories each, for each system.

\section{STATISTICAL FUNCTIONS}

The statistical functions which we use to characterize the local structure of the mixtures studied are the VP character- 
istics, the nearest neighbor radial distribution, and the three orientational order parameters. The distributions are defined in an unambiguous way, allowing the characterization of the local structure. The two approaches are well documented in our previous papers, ${ }^{36,38,44-46}$ therefore, we only summarize here the most important details, while the description of the last one will be given later. For a given distribution of a set of molecules, the VP of the central molecule is the region of space closer to this molecule than to any other one. ${ }^{47,48}$ The VP represents the volume element that belongs to this central molecule. Conversely the reciprocal volume of a $\mathrm{VP}, \rho=1 / V$, can be a measure of the local density around its central molecule. The local environment of the molecules can be characterized by the shape of the corresponding VP. To quantify this property, Ruocco et $a l .{ }^{48}$ proposed to use the asphericity parameter of the VP, $\eta$, defined as

$$
\eta=\frac{S^{3}}{36 \pi V^{2}}
$$

where $S$ is the total surface area of the VP. The value of $\eta$ is unity for a perfectly spherical VP, and it becomes larger for less spherical ones. The VP of the molecules has been determined using the algorithm of Ruocco $\mathrm{et} \mathrm{al.}{ }^{48}$ The distributions of DMSO and water molecules have been analyzed in detail by means of the VP. The oxygen atoms of water and the methyl groups of DMSO have been considered in this analysis as VP centers. ${ }^{37,38,49-51}$ In order to quantify in which manner the local ordering of water and DMSO molecules is affected by the increasing $X_{\mathrm{DMSO}}$, we have also calculated the VP distributions by disregarding one component and taking solely the other one into account in the analysis. ${ }^{22-24,51}$ The nearest neighbor radial distribution function is equivalent to the conventional radial distribution function; the only difference is that it is restricted now to the nearest neighbor molecules. Indeed, the total radial distribution function between $\alpha$ and $\beta$ atoms is resolved in terms of the first, second, $\ldots$, and the nth neighbor atom $\beta$ around $\alpha$. Based on these distributions, one can calculate the average position $\left\langle\mathrm{r}_{\alpha} \ldots \beta\right\rangle(n)$ and its corresponding fluctuation $\Delta \mathrm{r}_{\alpha} \ldots \beta$ for the nth first nearest neighbor $\beta$ atoms around a reference $\alpha$ atom. Its advantage over the conventional form is that the latter lacks a significant amount of details due to averaging over all molecules at a given distance range, while the nearest neighbor function monitors exclusively the nearest neighbor. This information is of great importance when we need to quantify the changes in the interactions between two molecules (or two sites), induced by the change in the mole fraction of one of the components. The basic idea is that a small value of the average distance between a central molecule and its nearest neighbor is an indication of the strength of their interaction, although one should have information on the orientation between the molecules in order to safely quantify that strength. Hydrogen bond geometry, $\mathrm{O}_{\mathrm{W}}-\mathrm{H}_{\mathrm{W}} \cdots \mathrm{X}$, involving water molecule can be described in terms of two hydrogen bond distances: the distance $\mathrm{r}_{\mathrm{H}_{W}} \ldots \mathrm{X}$, between the $\mathrm{H}$ atom of water and atom $X$, and the distance $\mathrm{r}_{\mathrm{O}_{W}} \ldots X$, between the oxygen atom of water and atom X. By applying the nearest neighbor approach, we calculated these distances as a function of $\mathrm{X}_{\mathrm{DMSO}}$.

\section{RESULTS AND DISCUSSIONS}

\section{A. Cluster distribution}

In order to get information on the local structure among DMSO molecules, we have analyzed the cluster size distribution of the DMSO molecules, constructed using distance criteria between their hydrophobic $\mathrm{CH}_{3}$ groups. A group of DMSO molecules form a cluster if each pair of DMSO molecules has one of their $\mathrm{CH}_{3}$ groups closer to each other than a threshold distance. The distance criterion is $X_{\mathrm{DMSO}}$ dependent and is associated with the average distance between a reference $\mathrm{CH}_{3}$ group and its first nearest $\mathrm{CH}_{3}$ neighbor on another molecule. These distances were calculated using the nearest neighbor approach and are displayed in Fig. 1. The cluster size distributions, $P(i)$, calculated at the studied mole fractions are shown in Fig. 2(a). As it is seen in this figure, the distribution is dominated by DMSO monomers $(i=0)$, whose contribution falls from $70 \%$ to $58 \%$ when $X_{\mathrm{DMSO}}$ is increased from 0.02 to 0.20 , while the contribution of dimers $(i=1)$ increases in this mole fraction range, and remains constant upon further increase of $X_{\text {DMSO }}$. The contribution of clusters of higher degree is weak and increases with $\mathrm{X}_{\mathrm{DMSO}}$ but remains always below 5\%. The distribution of the number of clusters is shown in Fig. 2(b). The number of cluster values is normalized by the total number of DMSO molecules in each mixture; thus, a value of 1.0 on the abscissa corresponds to a configuration where the number of clusters is equal to the number of DMSO molecules, i.e., all DMSO molecules exist as monomers. Conversely, values lower than 1 are indicative of a self-association of DMSO molecules. As is seen from Fig. 2(b), the average number of clusters and the corresponding fluctuation decrease drastically in the $X_{\mathrm{DMSO}}$ range between 0 and $0.10-0.12$, but this decrease becomes considerably smaller upon further increase of $X_{\text {DMSO }}$. These results suggest that the mixing scheme of DMSO with

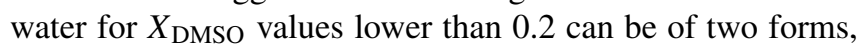
depending on the mixture composition. Thus, in the first case

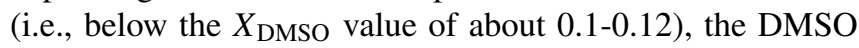
molecules are mainly solvated by water molecules, whereas in the second case, at higher DMSO mole fractions the solvation shell of DMSO contains both water and DMSO molecules. These results are in agreement with those obtained using the empirical potential Monte Carlo simulation to analyze the

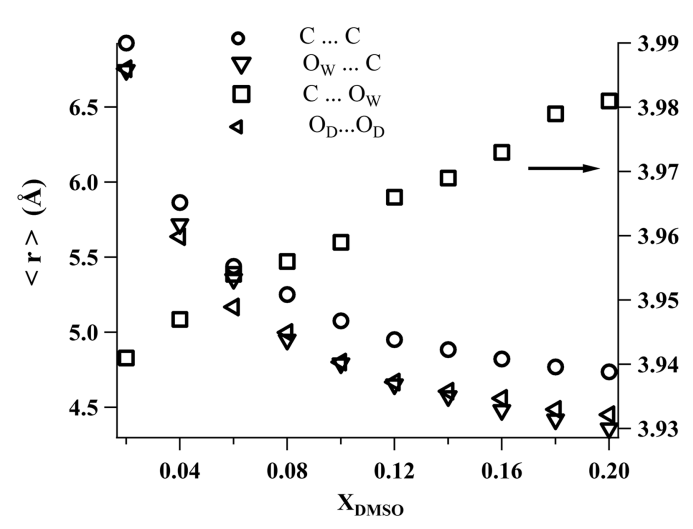

FIG. 1. Average distance between the nearest neighbor $\mathrm{C} \cdots \mathrm{C}, \mathrm{O}_{\mathrm{W}} \cdots \mathrm{C}$, $\mathrm{C} \cdots \mathrm{O}_{\mathrm{W}}$, and $\mathrm{O}_{\mathrm{D}} \cdots \mathrm{O}_{\mathrm{D}}$ atom pairs, calculated from the corresponding nearest neighbor radial distributions as a function of the DMSO mole fraction. 


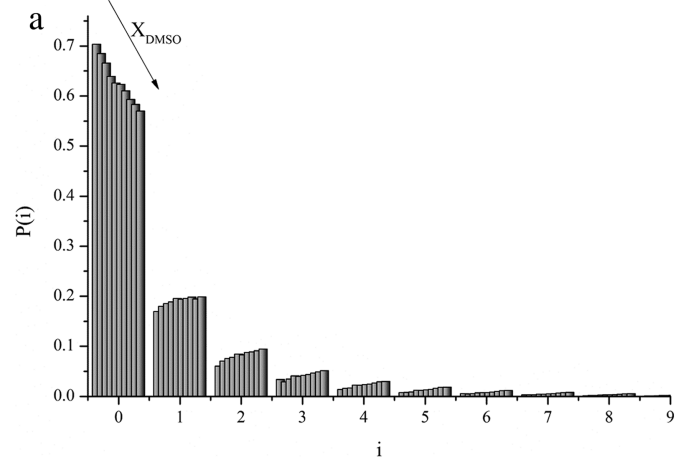

b

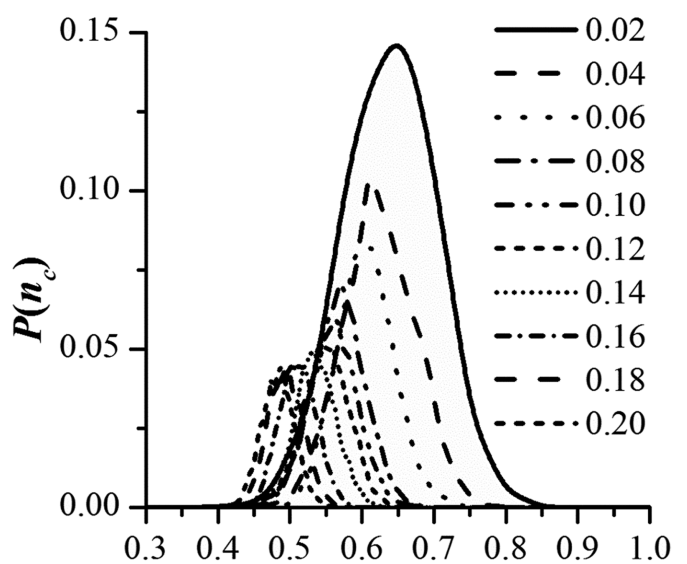

$\boldsymbol{n}_{c}$

FIG. 2. (a) The cluster size distribution of DMSO molecules, $P(i)$, constructed using a distance criterion between their hydrophobic $\mathrm{CH}_{3}$ groups. $i$ is the number of DMSO molecules in the cluster. A group of DMSO molecules form a cluster if each pair of DMSO molecules has at least one of their $\mathrm{CH}_{3}$ groups closer to each other than a threshold distance. The arrow indicates the increase of the $X_{\text {DMSO }}$ mole fraction from 0.02 to 0.20. (b) Distribution of the number of clusters normalized by the total number of DMSO molecules in the system, $n_{\mathrm{c}}$, at different DMSO mole fractions. Due to this normalization, the value of 1.0 on the abscissa corresponds to a configuration where all the DMSO molecules are monomers.

neutron scattering data on this mixture. ${ }^{52,53}$ They also are compatible with the ideas underlying the mean field model of this system. $^{54}$

\section{B. Density}

To develop a complementary understanding of the distribution of DMSO molecules in the mixture, an analysis through the VP was undertaken. In this analysis, the coordinates of the $\mathrm{CH}_{3}$ and $\mathrm{O}_{\mathrm{W}}$ atoms were considered. The distributions of the density of the VP, $P(\rho)$, are shown in Fig. 3(a). As is seen, adding DMSO to water induces an overall shift to lower local density values and, at the same time, leads to a rather complex shape of the distribution as compared to that in the case of neat water $\left(X_{\mathrm{DMSO}}=0.00\right)$. Indeed, the $P(\rho)$ distribution is unimodal in pure water, but already at $X_{\mathrm{DMSO}}=0.02$ a shoulder appears at the low density side of the main peak, which develops gradually to another peak at $X_{\mathrm{DMSO}}=0.20$. The intensity of this peak becomes slightly higher than that of the high density peak. Two other shoulders are observed at the lowest density side of $P(\rho)$ at $X_{\mathrm{DMSO}}=0.20$. The occurrence of these shoulders/peaks may correspond to molecules with
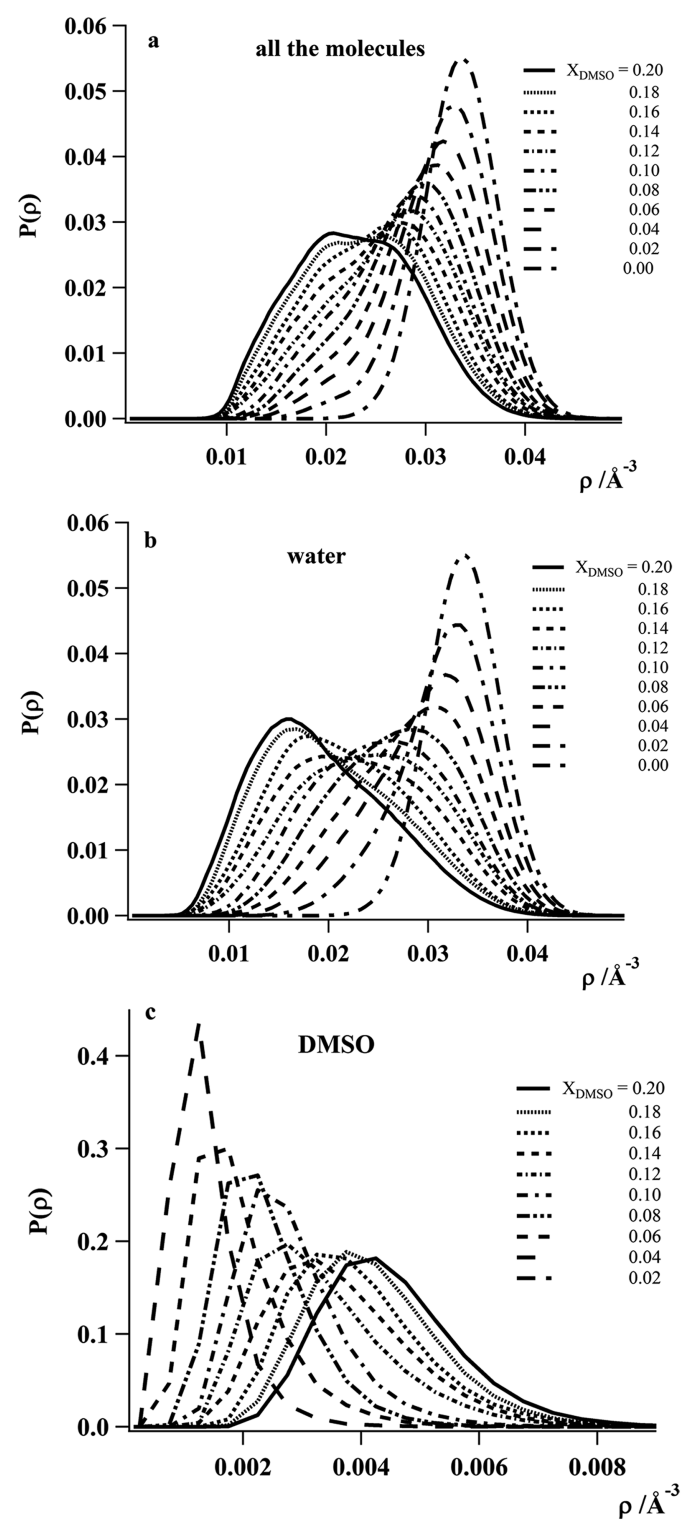

FIG. 3. Distribution of the density of the VP calculated by taking all the molecules into account (a), taking only the water molecules into account and disregarding DMSO (b), and taking only DMSO molecules into account and disregarding water (c) in the analysis.

markedly different local environments. Following our previous work, ${ }^{14}$ we may attribute the highest density peak to water molecules surrounded by other water molecules, while the shoulder located at the low density side of this peak, developing to a separate peak with increasing DMSO mole fraction, is associated with water molecules surrounded partly by DMSO molecules. The two other shoulders at low density values can be attributed to DMSO molecules surrounded partly by water molecules and to DMSO molecules surrounded solely by other DMSO molecules, respectively. ${ }^{37}$ To get more insight into the microscopic distribution of water and DMSO molecules, we have calculated the VP density distributions by disregarding one component and taking solely the other one into account in the analysis. Figure 3(b) shows the $P(\rho)$ distributions calculated by taking into account only water molecules and disregarding the DMSO molecules. As seen in this figure, there are two contributions: the highest density peak can be assigned 
to water molecules surrounded by other water molecules, while the lowest density peak can be assigned to water molecules having DMSO molecules in their local environment. Interestingly, in the range of $X_{\text {DMSO }}$ between 0.10 and 0.12 the intensity of the highest density peak becomes lower than that of the lowest density one, and they both shift gradually to lower density values. The same situation is mirrored in the behavior of the $P(\rho)$ distribution when only DMSO molecules are taken into account [see Fig. 3(c)]. Indeed, these distributions are mono-peaked at low and high $X_{\text {DMSO }}$ values, while they are characterized by the occurrence of two distinct contributions in the range of $X_{\mathrm{DMSO}}$ between 0.06 and 0.10 . The highest density peak is associated with DMSO molecules surrounded also by water molecules, while the lowest density peak is associated with DMSO molecules having only DMSO in their environment.

\section{Intermolecular voids}

The above presented findings can be further elaborated by calculating the distribution of the radius $R$ of the spherical vacancies in the systems of different compositions, as shown in Fig. 4(a). The radii of such spherical vacancies can simply be calculated by the VP analysis as the distances of the VP vertices from their central particles. ${ }^{14}$ The $P(R)$ distribution at $X_{\mathrm{DMSO}}=0.02$ is unimodal, and upon further increase of $X_{\mathrm{DMSO}}$ a shoulder develops at higher $R$ values, which becomes a well resolved contribution above $X_{\text {DMSO }}=0.1$. The low $R$ peak is associated with neat water-like domains, while the high $R$ peak can be associated with domains where water molecules have DMSO in their local environment. The inversion in the intensities of the two contributions occurs around $X_{\text {DMSO }}=0.12-0.14$. The $P(R)$ distribution obtained by disregarding the DMSO molecules and taking only water molecules into account [Fig. 4(b)] confirms this interpretation. Indeed, the position of the lower void radius peak does not seem to depend on $X_{\text {DMSO }}$, while that of the higher radius peak shifts gradually to larger $R$ values. When taking only the DMSO molecules into account and disregarding water molecules in the calculation of the $P(R)$ distributions [see Fig. 4(c)], the physical picture of the environment of DMSO molecules can be drawn as follows: at low $X_{\mathrm{DMSO}}$, the $P(R)$ distributions are unimodal at larger radii, corresponding to DMSO-devoid domains occupied by the disregarded water molecules, and indicate that DMSO is solvated by water molecules. For further increase of $X_{\mathrm{DMSO}}$, a clear transition in the shape of the $P(R)$ curve occurs at around 0.10-0.12. Indeed, at small side $R$ values, a shoulder emerges as a peak that shifts toward small void radii which characterizes the situation in neat DMSO, while the higher $R$ peak of $P(R)$ turns into a shoulder. The behavior of these two contributions as a function of $X_{\text {DMSO }}$ identifies the higher $R$ peak with voids indicative of domains of DMSO solvated by water molecules, whereas the lower- $R$ one corresponds to domains where DMSO molecules are present in the direct environment of another DMSO molecule.

\section{Asphericity}

The scheme of mixing DMSO with water can be further confirmed by analyzing the VP asphericity parameter distribution, whose behavior is illustrated in Fig. 5(a), including
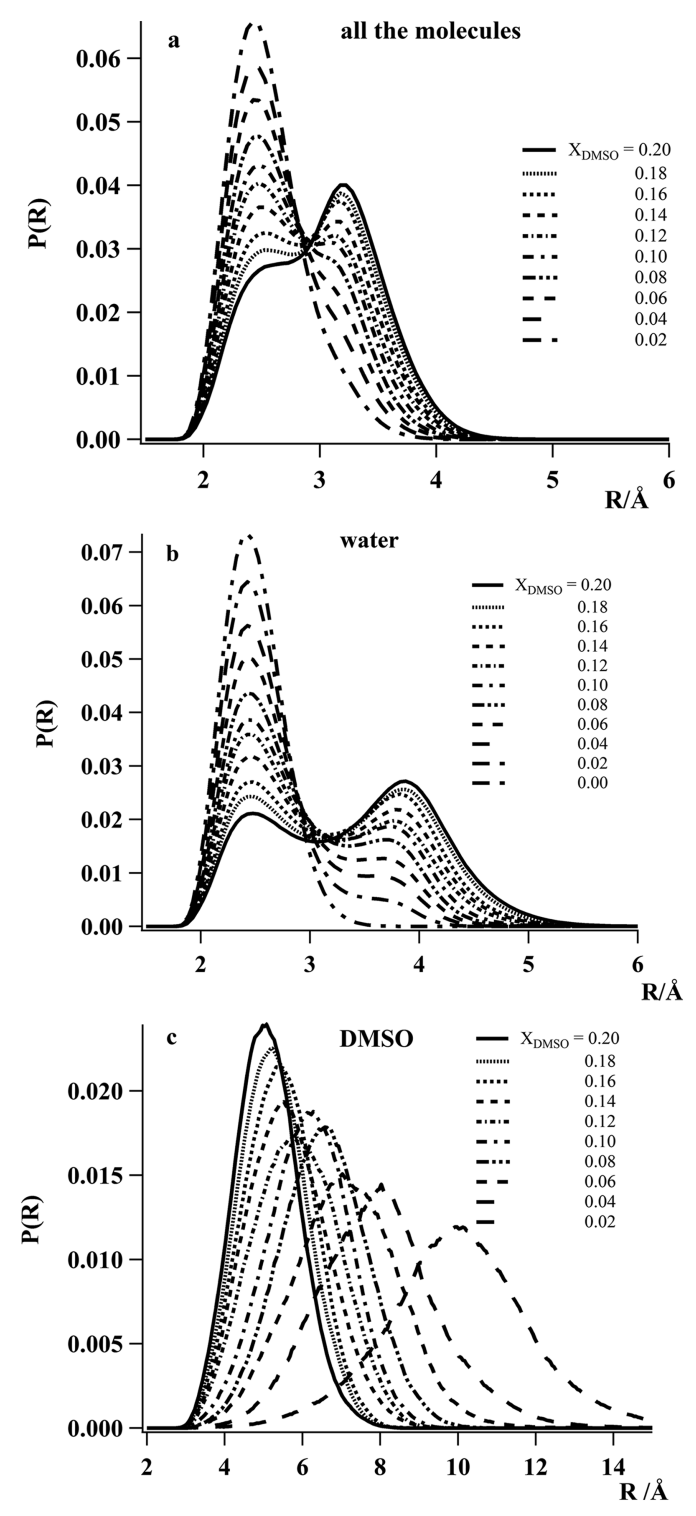

FIG. 4. Distribution of the radius of the spherical voids between the molecules in water-DMSO mixtures of different compositions, obtained by taking into account all the molecules (a), by taking only the water molecules into account and disregarding DMSO in the analysis (b), and by taking only the DMSO molecules into account and disregarding water in the analysis (c).

distributions in neat water and DMSO. When adding DMSO to water below the $X_{\text {DMSO }}$ threshold of 0.10-0.12, the asphericity parameter distribution calculated by taking only water molecules into account and disregarding DMSO molecules broadens noticeably, and the rate of broadening decreases upon further increase of $X_{\text {DMSO }}$. This indicates that the local environment of the central water molecule becomes less and less spherical due to the increasing number of DMSO molecules in their environment.

The behavior of the asphericity distribution, calculated when only DMSO molecules are taken into account and water molecules are disregarded, is shown in Fig. 5(b). The asphericity distributions show that the local environment around a reference DMSO molecule becomes more and more spherical with increasing DMSO mole fraction, which is consistent with the fact that more and more DMSO molecules are present in the local environment of a reference DMSO molecule. The 

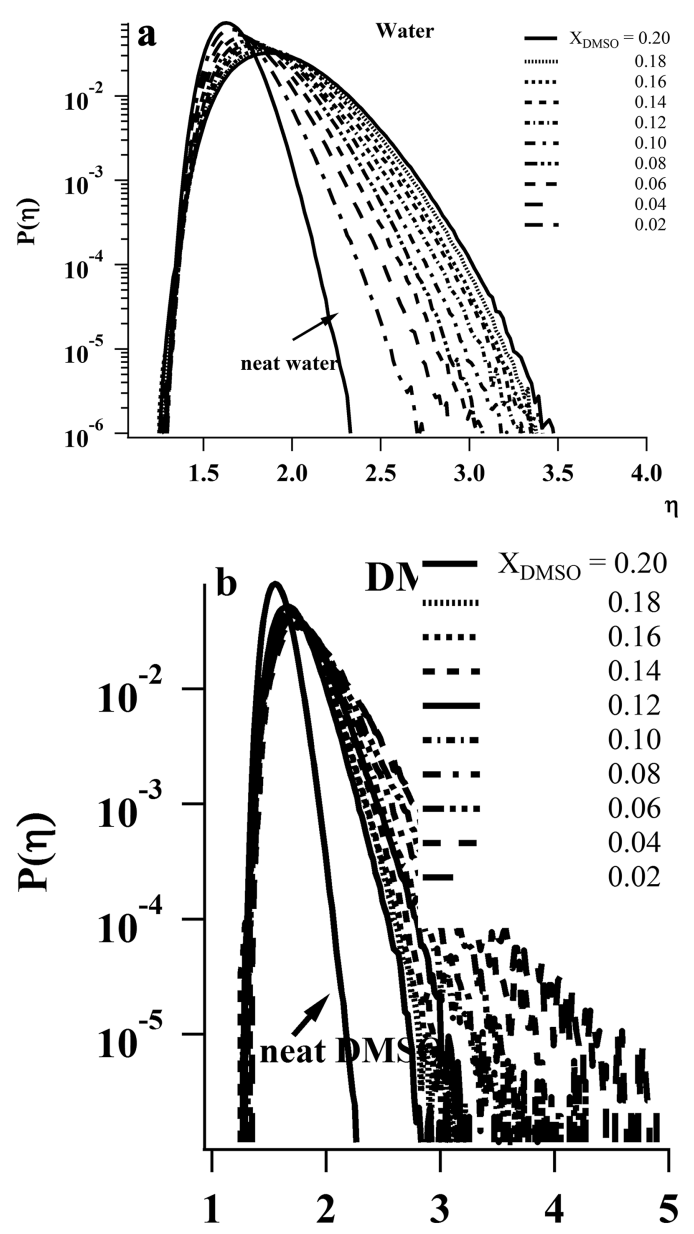

FIG. 5. Distribution of the asphericity parameter of the VP of the molecules in water-DMSO mixtures of different compositions by taking only the water molecules into account and disregarding DMSO in the analysis (a), and by taking only the DMSO molecules into account and disregarding water in the analysis (b). The distributions of the asphericity parameter of the VP in neat water and DMSO are given for comparison.

two distributions are in line with our previous finding that molecules having unlike molecules in their environment exist in a less spherical local environment than those surrounded by like neighbors only. ${ }^{37}$

\section{E. Free energy of mixing}

To see how the revealed changes of the local structure of the molecules occurring in the DMSO mole fraction range around 0.1 are reflected in the thermodynamic properties of the system, we have calculated the free energy of mixing the two components, $\Delta A^{\text {mix }}$, in the $X_{\text {DMSO }}$ range between 0 and 0.2 with an increment of 0.02 in the same way as described in our previous paper. ${ }^{36}$ The results are shown in Fig. 6. As can be seen, the free energy of mixing decreases steadily in this low DMSO mole fraction range, but the slope of this decrease becomes noticeably smaller around $X_{\text {DMSO }} \sim 0.1$. To demonstrate this, we have fitted straight lines to the data obtained in the DMSO mole fraction ranges of 0-0.08 and 0.1-0.2 and included these fitted lines in the figure. In light of our previous results, this finding indicates that the solvation of a new DMSO molecule by solely water neighbors corresponds to a larger free energy decrease than the solvation by neighbors of both types. This difference in the free

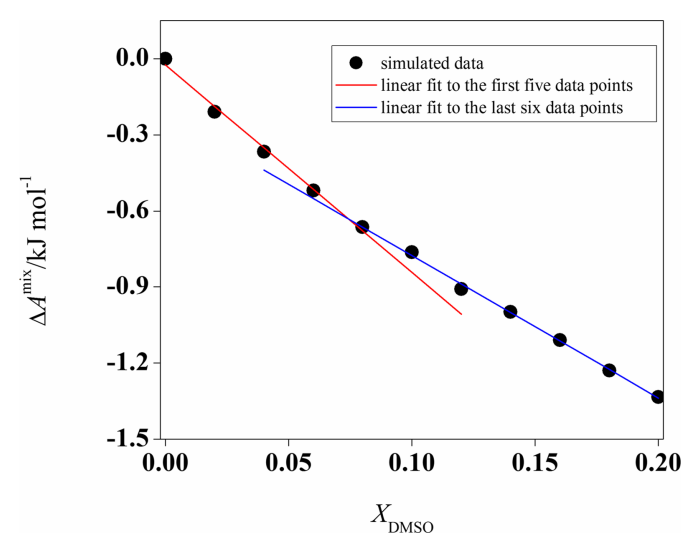

FIG. 6. Free energy of mixing of water and DMSO, as obtained from our simulations (filled circles). The straight lines fitted to the first five and last six data points, respectively, are shown in red and blue, respectively.

energy of solvation explains why DMSO molecules prefer to be surrounded solely by water neighbors if their mole fraction is small enough to allow such a mixing. On the other hand, above the DMSO mole fraction of about 0.1, the DMSO:water ratio in the system does not allow all DMSO molecules to be surrounded solely by water; thus, further increase of the DMSO mole fraction leads to the increase in the number of DMSO molecules that are in a mixed local environment rather than those being surrounded solely by water neighbors. These results are in agreement with the basic assumption of the model developed by Luzar to analyze the bulk free energy of mixing of the water/DMSO mixture. ${ }^{54}$ Indeed, in this model, only the hydrogen bond interactions among water molecules and between water and DMSO molecules were considered.

\section{F. Order parameter distribution of water}

During the process of adding DMSO to water, the local structure of water is obviously affected. Whether the DMSO molecules are ideally dissolved or not is determined by the balance of the competition between the original water structure and the hydration structure around the DMSO molecules, in particular, around the hydrophilic and hydrophobic groups. In this part of the paper, we want to address the issue of how the local structure of water is changed when adding DMSO to water. A large number of different geometric and order parameters have been used to characterize the local structure of water. ${ }^{55}$ We considered in our analysis three orientational order parameters $q_{3}, q_{4}$, and $q_{5}$.

The order parameter $q_{4}$ which probes the tetrahedral distribution of water was first proposed by Chau and Hardwick ${ }^{56}$ and later used by Errington and Debenedetti, ${ }^{57}$

$$
q_{4}=1-\frac{3}{8} \sum_{j=1}^{3} \sum_{k=j+1}^{4}\left(\cos \psi_{j k}+\frac{1}{3}\right)^{2},
$$

The order parameter $q_{3}$ probes the trigonal geometry of three coordinated water molecules while $q_{5}$ probes the trigonal bipyramidal geometry of five coordinated ones. They were proposed by Henchman and Cockram ${ }^{58}$ in order to take into account the non-tetrahedral coordination of water when analyzing the local structure. They provided in their 
paper experimental (X ray, neutron scattering, and Raman spectroscopy) and theoretical arguments in favor of considering the non-local tetrahedral distribution of water. These two orientational order parameters are given by the following equations:

$$
\begin{gathered}
q_{3}=1-\frac{4}{7} \sum_{j=1}^{3} \sum_{k=j+1}^{4}\left(\cos \psi_{j k}+\frac{1}{2}\right)^{2} \\
q_{5}=1-\frac{6}{35} \sum_{i=1}^{2} \sum_{i=j+1}^{3}\left(\cos \psi_{i j}+\frac{1}{2}\right)^{2}-\frac{3}{10} \sum_{i=1}^{3} \sum_{k=1}^{2}\left(\cos \psi_{i k}\right)^{2} \\
-\frac{3}{40}\left(\cos \psi_{k k}+1\right)^{2}
\end{gathered}
$$

where $\psi_{j k}$ is the angle between the axes connecting the oxygen atom of the central water molecule with those of its nearest neighbors (considering only the first four, three, and five ones, respectively). It should be stressed that $\mathrm{k}$ index in Eq. (5) describes the axial water molecules. The value of $q_{\mathrm{i}}(\mathrm{i}=3,4$, and 5) always falls between 0 and 1 ; if the local environment of the central molecule is perfectly trigonal, tetrahedral, and trigonal bipyramidal, the value of $q_{\mathrm{i}}$ ( $\mathrm{i}=3,4$, and 5) is unity, while more distorted local environments result in smaller $q_{\mathrm{i}}$ values. We first calculated these parameters for water and liquid argon systems. The main objective of this calculation is to identify the contribution of the close-packed (non-tetrahedral) local structure that characterizes the liquid Ar system $(\mathrm{T}=130 \mathrm{~K})$ and to help disentangle the contributions of the tetrahedral and the non-tetrahedral local structure that characterize the water system. The results are depicted in Fig. 7. Based on the comparison between the two systems, the low $q_{3}$ values in $f\left(q_{3}\right)$ of water is associated with a close-packed local structure (non-tetrahedral) while the highest $q_{3}$ values' contribution indicates that the nearest three water molecules to a central one adopt almost a perfect trigonal structure. ${ }^{58}$ The $f\left(q_{5}\right)$ distribution captures mainly the contribution of closepacked water molecules. The shoulder occurring at high $q_{5}$ values describes, in our point of view, the tetrahedral distribution of the four water molecules (among the first five neighbors). Similarly to what was found in previous studies, ${ }^{45,59-65}$ the tetrahedral order parameter distributions $f\left(q_{4}\right)$ of water

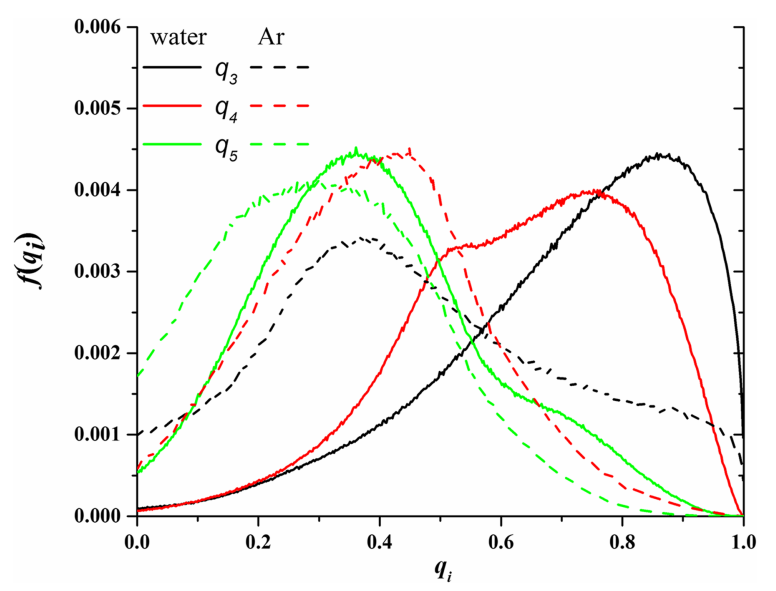

FIG. 7. Trigonal, tetrahedral, and trigonal bipyramidal distributions in pure water at room temperature and in liquid $\mathrm{Ar}$ at $\mathrm{T}=130 \mathrm{~K}$. exhibit two modes. The first one, located at high $q_{4}$ values, is associated with water molecules having a locally transient tetrahedral structure, while the second one, located at lower $q_{4}$ values, corresponds to deformed or non-tetrahedral local structures. The presence of this low $q_{4}$ contribution in $f\left(q_{4}\right)$ can be rationalized by considering that it is also present in the tetrahedral distribution of monoatomic systems, such as liquid Ar, having no specific interactions. This suggests that this contribution may be understood in terms of close-packed local structure.

The effect of adding DMSO molecules on the local structure of water is quantified by three situations associated with the choice of the atoms in the calculations of $q_{3}, q_{4}$, and $q_{5}$ order parameter distributions. The first ones, $f\left(q_{3}\right), f\left(q_{4}\right)$, and $f\left(q_{5}\right)$, were calculated by considering solely neighboring water oxygen atoms around the central water oxygen. The distributions $g\left(q_{3}\right), g\left(q_{4}\right)$, and $g\left(q_{5}\right)$ were calculated taking all oxygen atoms around the reference oxygen atom into account, regardless of the species to which they belong to. These distributions give information on the distribution of water molecules around the hydrophilic part of DMSO. Finally, $h\left(q_{3}\right), h\left(q_{4}\right)$, and $h\left(q_{5}\right)$ were calculated by taking into account both the oxygen atom of water neighbors and the methyl groups of DMSO neighbors of the central water molecule. These distributions give thus specific information on the local structure of water molecules including those having a methyl (hydrophobic) group in their neighborhood. The three types of distributions are given in Fig. 8, at various DMSO mole fractions. All the three descriptors of the local structure of water associated with three, four, and five coordinated water molecules are sensitive to the increase of $X_{D M S O}$. Indeed, in the case of $f\left(q_{4}\right), g\left(q_{4}\right)$, and $h\left(q_{4}\right)$ distributions, the intensity of the contribution located at high $q_{4}$ values decreases with increasing $X_{D M S O}$; however, the extent of this decrease is noticeably higher in the case of $h\left(q_{4}\right)$ distribution. The contribution of the lowest $q_{4}$ values shifts to lower values and its intensity decreases slightly and broadens in the case of $f\left(q_{4}\right)$. In the case of $g\left(q_{4}\right)$, it remains almost constant while it increases in the case of $h\left(q_{4}\right)$. The comparison between the intensity of the high and low $q_{4}$ values' contributions indicates that there is a turnover occurring around $\mathrm{X}_{\mathrm{DMSO}}=0.10-0.12$. This effect is more pronounced for water molecules that are in the direct environment of the methyl group of DMSO. The same trend is observed for the local structure of water as described by water molecules having three neighbors. Indeed, the intensity of the highest $q_{3}$ values' contribution in $f\left(q_{3}\right), g\left(q_{3}\right)$, and $h\left(q_{3}\right)$ decreases with increasing $\mathrm{X}_{\mathrm{DMSO}}$. The extent of this decrease is important in the case of $h\left(q_{3}\right)$. A contribution at low $q_{3}$ values emerges for $X_{D M S O}$ higher than $0.10-012$ and its intensity is the highest in the case of $h\left(q_{3}\right)$. In the case of five coordinated water molecules, the distributions $f\left(q_{5}\right), g\left(q_{5}\right)$, and $h\left(q_{5}\right)$ shift to lower $q_{5}$ values with a noticeable broadening in the case of $f\left(q_{5}\right)$. The intensity of the highest $q_{5}$ values decreases and vanishes starting from $X_{D M S O}=0.10-0.12$.

These distributions are calculated on the basis of distance criteria between a reference water molecule and its five first neighbors (including water as well as the hydrophilic and hydrophobic parts of DMSO). It is then possible to rationalize the behavior of these distributions by analyzing the average 

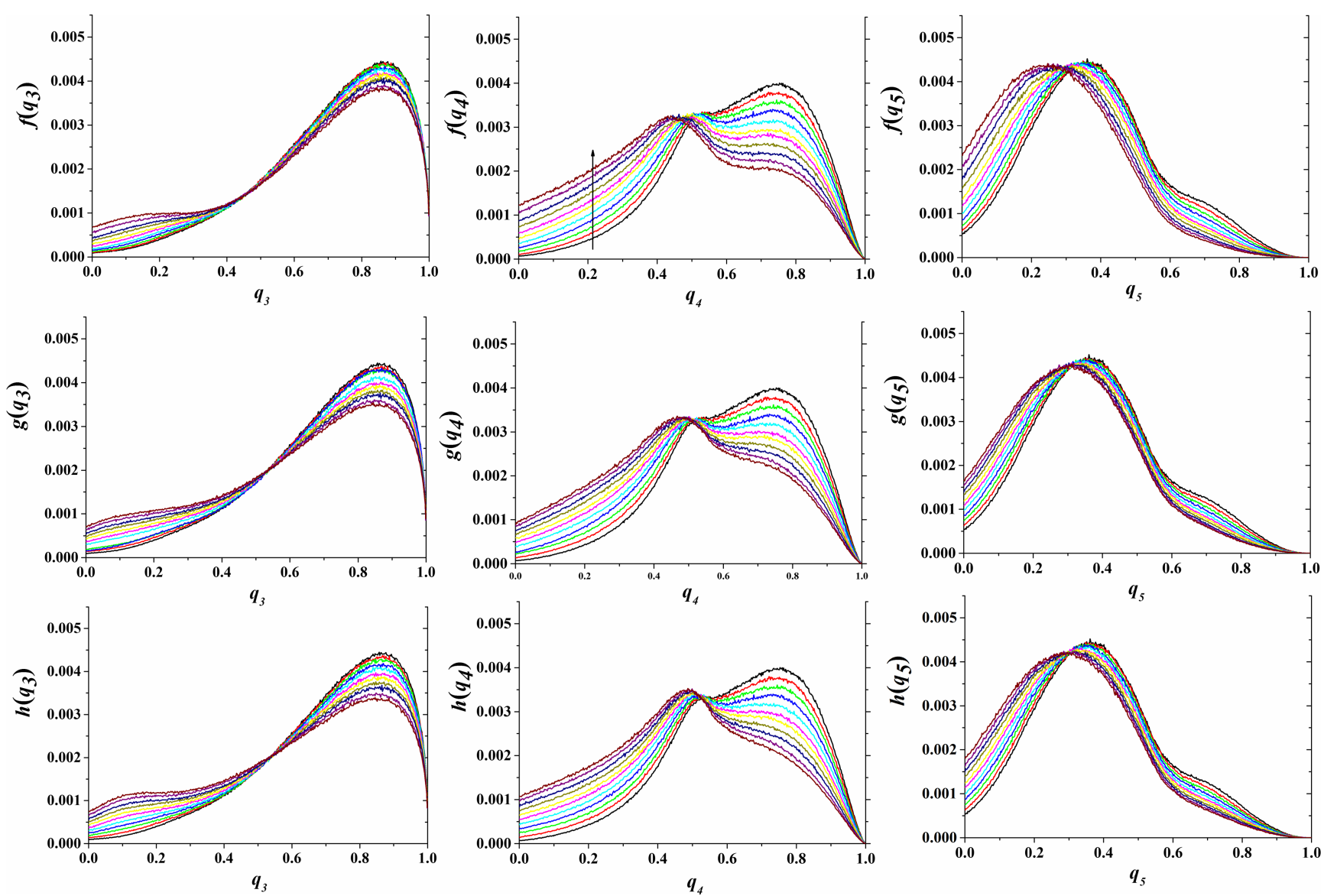

FIG. 8. Distributions of the orientational order parameters $q_{3}, q_{4}$, and $q_{5}$ describing the trigonal, tetrahedral and trigonal bipyramidal local distribution of water, respectively. In the first row, only oxygen atoms of water are taken into account. In the second row, these distributions are calculated for water molecules around the hydrophilic group of DMSO, namely, its oxygen atom while in the third row, they are calculated for those around the methyl hydrophobic group of DMSO. These functions were calculated as a function of $X_{D M S O}$.

distance characterizing the hydrogen bonding involving water molecules. Hydrogen bond geometry, $\mathrm{O}_{W}-\mathrm{H}_{W} \cdots X$, involving water molecules can be described in terms of two hydrogen bond distances: the distance $\mathrm{r}_{\mathrm{H}_{W}} \ldots \mathrm{X}$, between the $\mathrm{H}$ atom of water and atom $X$, and the distance $\mathrm{r}_{\mathrm{O}_{W}} \ldots X$, between the oxygen atom of water and atom $X$. These distances are illustrated in the inset of Fig. 7. When a linear hydrogen bond occurs between $\mathrm{O}_{W} \cdots X$, the distance between $\mathrm{O}_{W} \cdots X$ is equal to the sum of the distance between the intramolecular $\mathrm{O}_{W}-\mathrm{H}_{W}$ (that is equal to $0.96 \AA$ ) and the distance $\mathrm{H}_{W} \cdots X$. This situation corresponds to a strong hydrogen bond. While in the other cases, it corresponds to a bent hydrogen bond and then to a weak hydrogen bond. The behavior of the distance $\mathrm{r}_{\mathrm{O}_{W}} \cdots X$ as a function of $\mathrm{r}_{\mathrm{H}_{W}} \cdots X$ will allow comparing the hydrogen bond interactions between two water molecules, $\mathrm{X}$ is equal to $\mathrm{O}_{\mathrm{W}}$, between water and the hydrophilic group of DMSO, $\mathrm{X}$ is equal to $\mathrm{O}_{\mathrm{D}}$ (oxygen atom of DMSO), and between water and the methyl hydrophobic group of DMSO, $\mathrm{X}$ is equal to $\mathrm{C}$. These distances will be calculated for the five first neighbors of a reference water molecule and will achieve a high resolution in the description of the local structure around water molecules. The behavior of $\left\langle\mathrm{r}_{\mathrm{O}_{W}} \cdots X\right\rangle(n=1-5)$ as a function of $\left\langle\mathrm{r}_{\mathrm{H}_{W}} \cdots X\right\rangle(n=1-5)$ calculated at various $\mathrm{X}_{\mathrm{DMSO}}$ is illustrated in Fig. 7. The behavior of the distance characteristics between a reference water molecule and its five first neighbor water molecules indicates that the hydrogen bond geometry is almost linear, moderately bent for the first and the second, respectively. The third, fourth, and fifth neighbors are characterized by an orientational disorder (strongly bent hydrogen bonding geometry). This evidences that the hydrogen bonds between a reference water molecule and its five first nearest neighbors are not equivalent. The two first neighbors have strong hydrogen bonding with a reference water molecule while the third, fourth, and fifth neighbors are characterized by disordered (bent) hydrogen bonding and may be described as close-packed neighbors. Adding DMSO to water mainly reinforces the close-packed structure of these neighbors while it has almost no effect on the hydrogen bonding involving the first neighbor. However, it has a strong effect on the hydrogen bonding characteristic distances between water and its second neighbor by making the hydrogen bonding more and more bent.

The comparison between the hydrogen bonding characteristic distances between water molecules and those between water and the hydrophilic group of DMSO (oxygen atom $\mathrm{O}_{\mathrm{D}}$ ) shows that the hydrogen bonding is the strongest in the latter case. These results are in agreement with the previously reported neutron scattering, ${ }^{52,53} \mathrm{NMR},{ }^{66}$ and MD simulations ${ }^{67}$ as well as with the ideas underlying the mean field model of this system. ${ }^{54}$ This ranking is true for the first and 
second neighbor DMSO molecules of a reference water one. Adding DMSO to water does not affect the former one while it weakens the latter one by increasing the characteristic distances and maintaining the linear geometry of the hydrogen bonding. The hydrogen bonding characteristic distance involving the third neighbor DMSO to a reference water molecule is sensitive to the increase of $X_{D M S O}$. Indeed, the geometry becomes more and more bent. The fourth and fifth hydrophilic DMSO neighbors are characterized by strongly bent hydrogen bond geometry compatible with a close-packed local structure.

The hydrogen bonding distance characteristics between water and the hydrophobic methyl group of DMSO indicates a strongly bent geometry. This result is in accordance with previous findings using empirical potential Monte Carlo simulation used to interpret the neutron scattering experiment ${ }^{52,53}$ on higher $X_{D M S O}$ mole fractions than those studied in the present paper. Adding DMSO to water does not affect drastically the hydrogen bond distance characteristics of the first five methyl group neighbors to a reference water molecule. Indeed, the geometry remains mainly strongly bent which is compatible with a close-packed structure.

The behavior of the hydrogen bond characteristic distances characterizing the local environment of water molecules may be correlated with the behavior of the trigonal, tetrahedral, and trigonal bipyramidal distributions. Indeed, as it is shown in Fig. 7, the comparison between the distance threshold that was used in the calculation of these distributions and the hydrogen bonding characteristic distances clearly indicates that (i) in the case of the trigonal distribution involving only water neighbor molecules, it may be correlated with the increase of the bent hydrogen bond geometry involving the second neighbor, (ii) in the case of the tetrahedral distribution, the decrease in the intensity of the high $q_{4}$ values is associated, in our opinion, with the behavior of the second neighbor while the increase of the lowest $q_{4}$ values is associated with the weakening of the hydrogen bonding (bent geometry) between a reference water molecule and its third and fourth nearest neighbors in favor of a close-packed local structure. It should be noticed that in many previous works, it was pointed out that the high $q_{4}$ values are associated with the fact that the hydrogen bond network of water is not perfect but contains defects characterized geometrically by the presence of the fifth molecule in the first coordination shell. ${ }^{59,68-71}$ The extent of the variation of the high and low $q_{4}$ values is higher for water molecules near the hydrophobic group (Fig. 9). The shape of the trigonal bipyramidal distribution is mainly dominated by the behavior of the third, fourth, and fifth neighbors that have a strong bent geometry and may be described as a close-packed local structure.

In order to go further in the interpretation of the characteristic distances of the hydrogen bonding involving water molecules, we analyzed the behavior of the fluctuation $\Delta \mathrm{r}_{\mathrm{O}_{W}} \ldots \mathrm{O}_{W}(n)$ associated with the average distance $\left\langle\mathrm{r}_{\mathrm{O}_{W}} \ldots \mathrm{O}_{W}\right\rangle(n)$, which is illustrated in Fig. 10. In pure water, $\Delta \mathrm{r}_{\mathrm{O}_{W}} \ldots \mathrm{O}_{W}(n)$ goes through a maximum at the fifth nearest neighbor, whose position at $r=3.4 \AA$ coincides with that of the first minimum of $g_{\mathrm{O}_{W}} \ldots \mathrm{O}_{W}(r)$. The occurrence of a maximum in the radial fluctuation of molecules located at the boundary

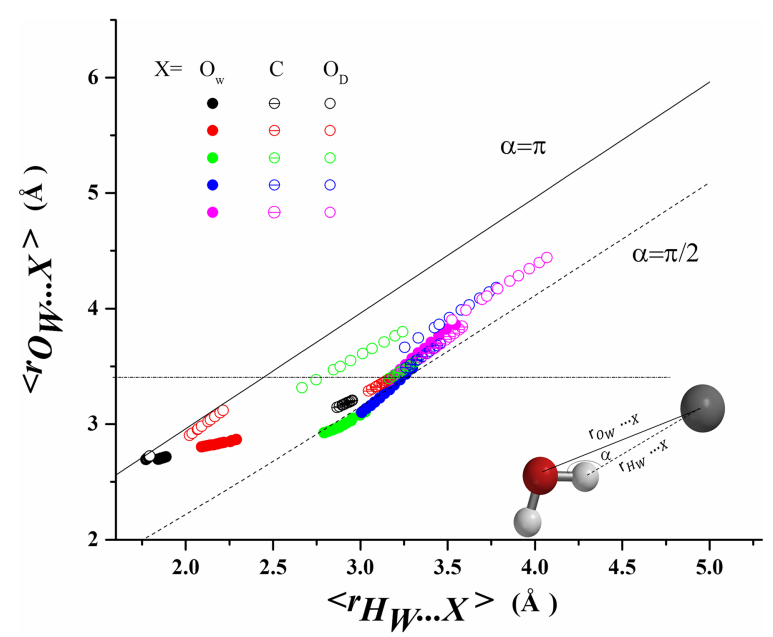

FIG. 9. The behavior of $\left\langle\mathrm{r}_{\mathrm{O}_{W}} \cdots X\right\rangle(n=1-5)$ as a function of $\left\langle\mathrm{r}_{\mathrm{H}_{W}} \cdots X\right\rangle$ $(n=1-5)$ calculated at various $\mathrm{X}_{\mathrm{DMSO}}$. These hydrogen bonding characteristic distances are between water molecules (Ow), between water molecules and the hydrophilic group $\mathrm{O}_{\mathrm{D}}$ of DMSO, and between water molecules and the hydrophobic group C. These distances were calculated for the five first nearest neighbors of water $(n=1-5) . \alpha=0$ corresponds to the situation where these distances are compatible with a linear geometry of the hydrogen bonding while $\alpha=\pi / 2$ corresponds to a strongly bent geometry. The horizontal line indicates the threshold distance $3.4 \AA$ used in the calculation of the trigonal, tetrahedral, and bipyramidal distributions.

of the first coordination shell is not only specific for water, but it also occurs for monoatomic systems, such as $\mathrm{Ar}^{46}$ as well as for systems of apolar molecules, such as $\mathrm{CO}_{2} \cdot{ }^{44}$ Both $\Delta \mathrm{r}_{\mathrm{O}_{W}} \ldots \mathrm{O}_{W}$ and the average distance $\left\langle\mathrm{r}_{\mathrm{O}_{W}} \ldots \mathrm{O}_{W}\right\rangle$ of the first nearest water neighbor are slightly affected by adding DMSO, while they are moderately affected for the second neighbor. However these parameters are drastically affected for the third, fourth, and fifth nearest water neighbors. One may notice that the maximum of the fluctuation $\Delta \mathrm{r}_{\mathrm{O}_{W}} \cdots \mathrm{O}_{W}$ is shifted gradually from the fifth to the fourth nearest neighbor and the turnover occurs at $X_{D M S O}$ between 0.10 and 0.16 . These results point out to the fact that DMSO affects the local structure of water by mainly increasing the distance and the fluctuation of the fifth,

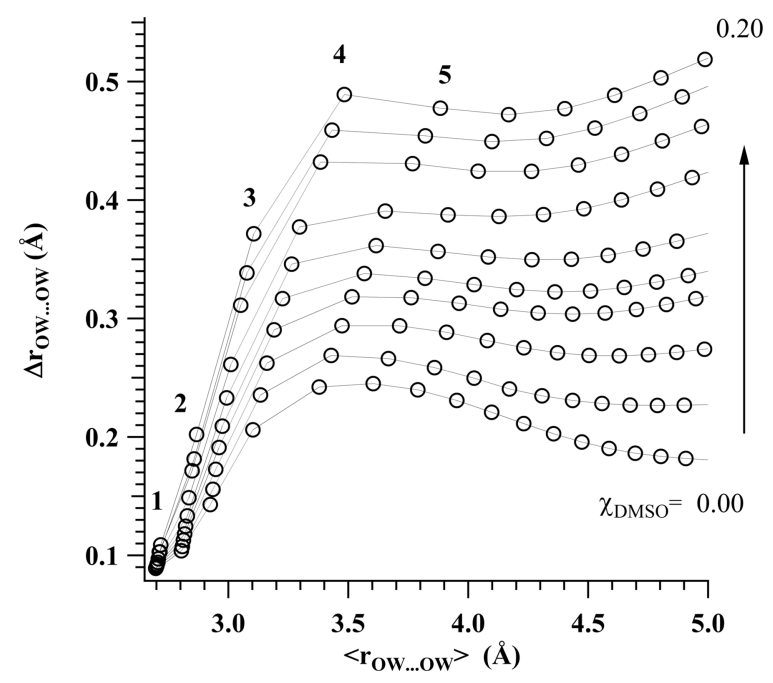

FIG. 10. The behavior of the distance fluctuation $\Delta \mathrm{r}_{\mathrm{O}_{W}} \cdots X(n)$ as a function of the corresponding average distance, $\left\langle\mathrm{r}_{\mathrm{O}_{W}} \cdots \mathrm{O}_{W}\right\rangle(n)$, between a reference oxygen atom of water and its nth neighbors. 
fourth, and third neighbor water molecules of a reference one. While the first and the second nearest neighbor are slightly affected by increasing $X_{D M S O}$.

\section{CONCLUSIONS}

In this paper, we studied the mixing scheme of DMSO with water in the water-rich composition range, namely, at DMSO mole fractions between 0.0 and 0.20 . Statistical functions such as the nearest neighbor radial distribution functions, distributions of several characteristics of the VP around a reference atom, the DMSO cluster size distributions, and the trigonal, tetrahedral, and trigonal bipyramidal orientational order parameter distributions of the water molecules were calculated, among water molecules as well as around the hydrophobic and the hydrophilic groups of DMSO. The value of the average distance between two DMSO molecules for

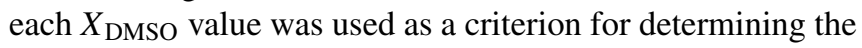
cluster size distribution of DMSO molecules. These distributions show that DMSO molecules exist mainly in a monomer state and do not show any percolating behavior. The analysis of the density, the spherical void radius, and the asphericity of the VP distributions determined by considering only one of the components and disregarding the other one reveals that

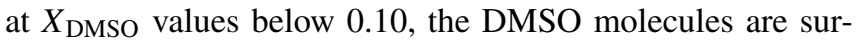
rounded by water molecules, while upon further increase of $X_{\mathrm{DMSO}}$, both water and DMSO molecules are present around a reference DMSO molecule. Correspondingly, the free energy of mixing DMSO and water exhibits less steep composition dependence above this DMSO mole fraction than below it. The analysis of the trigonal, tetrahedral, and trigonal bipyramidal distributions of water molecules indicates that adding DMSO induces in general a distortion of these orientations while in favor of a close-packed local structure. The turnover between the two local structures occurs in $X_{\text {DMSO }}$ around $0.10-0.12$. At a given $X_{\mathrm{DMSO}}$, water is more ordered in the vicinity of the hydrophilic group of DMSO while it is more closely packed near the hydrophobic one. These changes in the local structure of the DMSO-water mixture at around 0.10.12 are in accordance with the mass spectroscopy analysis. ${ }^{20}$ Our findings also correlate with both the low rate of increase of adsorption of DMSO on cellulose at low DMSO mole fractions below 0.1-0.12 and the change in the solvation of protein from preferential hydration at low DMSO mole fractions to preferential DMSO binding at higher DMSO mole fractions than 0.1-0.12. Furthermore, the spectroscopic properties are expected to be affected by the DMSO-water hydrogen bond interactions below $0.1-0.12$; however, they are also affected by dipole-dipole interactions between DMSO molecules.

\section{ACKNOWLEDGMENTS}

The Center de Ressources Informatiques (CRI) de l'Université de Lille and Center Régional Informatique et d'Applications Numériques de Normandie (CRIANN) are gratefully acknowledged for the CPU time allocation. The study was partly supported by Dean's Grant for Progressive Research Projects from Saga University (Japan) allowing
B.A.M., A.I., and T.T. to discuss the results at Saga University. A.I., B.A.M., and P.J. acknowledge the financial support of the Hungarian-French Intergovernmental Science and Technology Program (PHC Balaton 2016) under Project Nos. 36402ND (France) and TÉT_15-1-2016-0029 (Hungary). P.J. acknowledges the financial support of the Hungarian NKFIH Foundation under Project No. 119732.

${ }^{1}$ M. I. Voronova, T. N. Lebedeva, M. V. Radugin, O. V. Surov, A. N. Prusov, and A. G. Zakharov, J. Mol. Liq. 126(1-3), 124-129 (2006).

${ }^{2}$ T. Arakawa, Y. Kita, and S. N. Timasheff, Biophys. Chem. 131(1-3), 62-70 (2007).

${ }^{3}$ A. L. Fink and A. I. Ahmed, Nature 263(5575), 294-297 (1976).

${ }^{4}$ A. Huang, C. Liu, L. Ma, Z. Tong, and R. Lin, J. Chem. Thermodyn. 49, 95-103 (2012).

${ }^{5}$ P. Westh, J. Phys. Chem. 98(12), 3222-3225 (1994).

${ }^{6}$ J. T. W. Lai, F. W. Lau, D. Robb, P. Westh, G. Nielsen, C. Trandum, A. Hvidt, and Y. Koga, J. Solution Chem. 24(1), 89-102 (1995).

${ }^{7}$ Y. Koga, Solution Thermodynamics and Its Application to Aqueous Solutions (Elsevier, Amsterdam, 2007), pp. 175-203.

${ }^{8}$ J. J. M. Lindberg and M. Corrado, Acta Chem. Scand. 17, 1477-1478 (1963).

${ }^{9}$ D. D. Macdonald, M. D. Smith, and J. B. Hyne, Can. J. Chem. 49(17), 2817-2821 (1971).

${ }^{10}$ C. De Visser, W. J. M. Heuvelsland, L. A. Dunn, and G. Somsen, J. Chem. Soc., Faraday Trans. 1 74(0), 1159-1169 (1978).

${ }^{11}$ F. Rallo, F. Rodante, and P. Silvestroni, Thermochim. Acta 1(4), 311-316 (1970).

${ }^{12}$ Z. S. Klemenkova and E. G. Kononova, J. Solution Chem. 44(2), 280-292 (2015).

${ }^{13}$ Y. Koga, Y. Kasahara, K. Yoshino, and K. Nishikawa, J. Solution Chem. 30(10), 885-893 (2001).

${ }^{14}$ S. A. Markarian and A. M. Terzyan, J. Chem. Eng. Data 52(5), 1704-1709 (2007).

${ }^{15}$ V. M. Wallace, N. R. Dhumal, F. M. Zehentbauer, H. J. Kim, and J. Kiefer, J. Phys. Chem. B 119(46), 14780-14789 (2015).

${ }^{16} \mathrm{~K}$. Mizuno, S. Imafuji, T. Ochi, T. Ohta, and S. Maeda, J. Phys. Chem. B 104(47), 11001-11005 (2000).

${ }^{17}$ Z. Lu, E. Manias, D. D. Macdonald, and M. Lanagan, J. Phys. Chem. A 113(44), 12207-12214 (2009).

${ }^{18}$ S. Lotze, C. C. M. Groot, C. Vennehaug, and H. J. Bakker, J. Phys. Chem. B 119(16), 5228-5239 (2015).

${ }^{19}$ N. Engel, K. Atak, K. M. Lange, M. Gotz, M. Soldatov, R. Golnak, E. Suljoti, J.-E. Rubensson, and E. F. Aziz, J. Phys. Chem. Lett. 3(24), 3697-3701 (2012).

${ }^{20}$ D. N. Shin, J. W. Wijnen, J. B. F. N. Engberts, and A. Wakisaka, J. Phys. Chem. B 105(29), 6759-6762 (2001).

${ }^{21}$ M. Chalaris and J. Samios, J. Mol. Liq. 98-99, 401-411 (2002).

${ }^{22}$ H. J. C. Berebdsen, J. P. M. Postma, W. F. van Gusteren, and J. Hermans, Intermolecular Forces (B. Pullman, Dordrecht, 1981).

${ }^{23}$ A. Luzar and D. Chandler, J. Chem. Phys. 98(10), 8160-8173 (1993).

${ }^{24}$ R. L. Mancera, M. Chalaris, K. Refson, and J. Samios, Phys. Chem. Chem. Phys. 6(1), 94-102 (2004).

${ }^{25}$ W. L. Jorgensen, J. Chandrasekhar, J. D. Madura, R. W. Impey, and M. L. Klein, J. Chem. Phys. 79(2), 926-935 (1983).

${ }^{26}$ P. A. Bopp, J. Samios, and M. D. Zeidler, J. Mol. Liq. 110(1-3), 1-2 (2004).

${ }^{27}$ C. Oostenbrink, A. Villa, A. E. Mark, and W. F. Van Gunsteren, J. Comput. Chem. 25(13), 1656-1676 (2004).

${ }^{28}$ H. J. C. Berendsen, J. R. Grigera, and T. P. Straatsma, J. Phys. Chem. 91(24), 6269-6271 (1987).

${ }^{29}$ S. Roy, S. Banerjee, N. Biyani, B. Jana, and B. Bagchi, J. Phys. Chem. B 115(4), 685-692 (2011).

${ }^{30}$ J. Gujt, E. Cázares Vargas, L. Pusztai, and O. Pizio, J. Mol. Liq. 228, 71-80 (2017).

${ }^{31}$ A. Idrissi, I. Vyalov, M. Kiselev, and P. Jedlovszky, Phys. Chem. Chem. Phys. 13(36), 16272-16281 (2011).

${ }^{32}$ A. Pinke and P. Jedlovszky, J. Phys. Chem. B 116(20), 5977-5984 (2012).

${ }^{33}$ P. Jedlovszky, A. Idrissi, and G. Jancsó, J. Chem. Phys. 130(12), 124516 (2009).

${ }^{34}$ A. Idrissi, R. D. Oparin, S. P. Krishtal, S. V. Krupin, E. A. Vorobiev, A. I. Frolov, L. Dubois, and M. G. Kiselev, Faraday Discuss. 167(0), 239-262 (2013). 
${ }^{35}$ A. Vishnyakov, A. P. Lyubartsev, and A. Laaksonen, J. Phys. Chem. A 105(10), 1702-1710 (2001).

${ }^{36}$ A. Idrissi, B. Marekha, M. Barj, and P. Jedlovszky, J. Phys. Chem. B 118(29), 8724-8733 (2014).

${ }^{37}$ A. Idrissi, B. Marekha, M. Kiselev, and P. Jedlovszky, Phys. Chem. Chem. Phys. 17(5), 3470-3481 (2015).

${ }^{38}$ A. Idrissi, K. Polok, W. Gadomski, I. Vyalov, A. Agapov, M. Kiselev, M. Barj, and P. Jedlovszky, Phys. Chem. Chem. Phys. 14(17), 5979-5987 (2012).

${ }^{39}$ A. Idrissi, K. Polok, M. Barj, B. Marekha, M. Kiselev, and P. Jedlovszky, J. Phys. Chem. B 117(50), 16157-16164 (2013).

${ }^{40}$ W. Smith and T. R. Forester, J. Mol. Graphics 14(3), 136-141 (1996).

${ }^{41}$ H. J. C. Berendsen, J. P. M. Postma, W. F. van Gunsteren, A. DiNola, and J. R. Haak, J. Chem. Phys. 81(8), 3684-3690 (1984).

${ }^{42}$ S. W. de Leeuw, J. W. Perram, and E. R. Smith, Proc. R. Soc. A 373(1752), 27-56 (1980).

${ }^{43}$ P. P. Ewald, Ann. Phys. 369(3), 253-287 (1921).

${ }^{44}$ A. Idrissi, P. Damay, and M. Kiselev, Chem. Phys. 332(1), 139-143 (2007).

${ }^{45}$ A. Idrissi, M. Gerard, P. Damay, M. Kiselev, Y. Puhovsky, E. Cinar, P. Lagant, and G. Vergoten, J. Phys. Chem. B 114(13), 4731-4738 (2010).

${ }^{46}$ I. Vyalov, M. Kiselev, T. Tassaing, J. C. Soetens, and A. Idrissi, J. Phys. Chem. B 114(46), 15003-15010 (2010).

${ }^{47}$ B. Okabe, K. Boots, S. Sugihara, and S. N. Chiu, Spatial Tessellations Concepts and Applications of Voronoi Diagrams (John Wiley, Chichester, 2000).

${ }^{48}$ G. Ruocco, M. Sampoli, A. Torcini, and R. Vallauri, J. Chem. Phys. 99(10), 8095-8104 (1993).

${ }^{49}$ A. Baranyai and I. Ruff, J. Chem. Phys. 85(1), 365-373 (1986).

${ }^{50}$ P. Jedlovszky, J. Chem. Phys. 111(13), 5975-5985 (1999).

${ }^{51}$ A. Idrissi, P. Damay, K. Yukichi, and P. Jedlovszky, J. Chem. Phys. 129(16), 164512 (2008).

${ }^{52}$ A. K. Soper and A. Luzar, J. Chem. Phys. 97(2), 1320-1331 (1992).
${ }^{53}$ A. K. Soper and A. Luzar, J. Phys. Chem. 100(4), 1357-1367 (1996).

${ }^{54}$ A. Luzar, J. Chem. Phys. 91(6), 3603-3613 (1989).

${ }^{55}$ E. Duboué-Dijon and D. Laage, J. Phys. Chem. B 119(26), 8406-8418 (2015).

${ }^{56}$ P. L. Chau and A. J. Hardwick, Mol. Phys. 93(3), 511-518 (1998).

${ }^{57}$ J. R. Errington and P. G. Debenedetti, Nature 409(6818), 318-321 (2001).

${ }^{58}$ R. H. Henchman and S. J. Cockram, Faraday Discuss. 167(0), 529-550 (2013).

${ }^{59}$ D. Bandyopadhyay, S. Mohan, S. K. Ghosh, and N. Choudhury, J. Phys. Chem. B 117(29), 8831-8843 (2013).

${ }^{60}$ D. Bandyopadhyay, S. Mohan, S. K. Ghosh, and N. Choudhury, J. Phys. Chem. B 118(40), 11757-11768 (2014)

${ }^{61}$ C. Branca, S. Maccarrone, S. Magazù, G. Maisano, S. M. Bennington, and J. Taylor, J. Chem. Phys. 122(17), 174513 (2005).

${ }^{62}$ M. D. Elola and B. M. Ladanyi, J. Chem. Phys. 125(18), 184506 (2006).

${ }^{63}$ S. Paul and S. Paul, J. Mol. Liq. 211, 986-999 (2015).

${ }^{64}$ R. Politi, L. Sapir, and D. Harries, J. Phys. Chem. A 113(26), 7548-7555 (2009).

${ }^{65}$ I. Skarmoutsos, M. Masia, and E. Guardia, Chem. Phys. Lett. 648, 102-108 (2016).

${ }^{66}$ R. Ludwig, T. C. Farrar, and M. D. Zeidler, J. Phys. Chem. 98(27), 6684-6687 (1994).

${ }^{67}$ S. Velaga, R. Berger, and J. Carlfors, Pharm. Res. 19(10), 1564-1571 (2002).

${ }^{68}$ F. Sciortino, A. Geiger, and H. E. Stanley, Nature 354(6350), 218-221 (1991).

${ }^{69}$ A. M. Saitta, T. Strässle, G. Rousse, G. Hamel, S. Klotz, R. J. Nelmes, and J. S. Loveday, J. Chem. Phys. 121(17), 8430-8434 (2004).

${ }^{70}$ T. Strässle, A. M. Saitta, Y. L. Godec, G. Hamel, S. Klotz, J. S. Loveday, and R. J. Nelmes, Phys. Rev. Lett. 96(6), 067801 (2006).

${ }^{71}$ A. Idrissi, I. Vyalov, N. Georgi, and M. Kiselev, J. Phys. Chem. B 117(40), 12184-12188 (2013). 